\title{
The Contralateral Ear in Unilateral Chronic Otitis Media - Does it Need Reckoning?
}

\author{
Ramakrishnan Narayanaswamy, ${ }^{1}$ Gaurav Awasthi ${ }^{1}$
}

\begin{abstract}
Introduction Chronic Otitis Media (COM) and compare it with a control group of healthy subjects.

\section{Materials and Methods} in the contralateral ears were assessed statistically for significance.

$\underline{\text { Results }}$

\section{Conclusion} disease harboring silently.

Keywords

Otitis Media, Unilateral; Contralateral Ear; Retraction; Myringosclerosis
\end{abstract}

\section{ABSTRACT}

This comparative study aims to analyze findings and their significance in the contralateral ears of patients with unilateral

One hundred patients of unilateral COM and 100 healthy individuals were included in this study from Jan 2015 to July 2016. The findings were noted in the contralateral ears of patients with unilateral COM and 100 control subjects. The changes found

In 100 cases of unilateral COM, 58 patients had some abnormality in the contralateral ear. Myringosclerosis patches being the most common finding were seen in 29 patients followed by TM retractions seen in 28 patients. Prevalence of abnormal findings was found to be significantly higher in the squamous group as compared to the mucosal group. In 100 ears in the control group, only $11 \%$ had positive findings. TM retractions were seen in 5 subjects (3 Attic and 2 Pars tensa retractions).

It can be statistically concluded that the disease of COM in one ear affects the contralateral ear slowly and silently. Hence both ears are affected as pairs and a slow pathological process continues behind an intact TM and kept hidden from the view unless it is actively sought for. Contralateral ears should also be actively followed up, in cases of unilateral COM in order to pick up

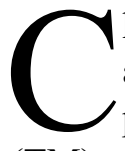
hronic Otitis Media (COM) is characterised as an inflammatory condition associated with a persisting perforation of the Tympanic membrane (TM) and chronic otorrhoea for more than 3 months. Histopathologically, COM is an inflammatory process of middle ear associated with irreversible tissue changes. This inflammatory process may be present even though the TM is intact and otorrhoea is absent - called as Silent Chronic Otitis Media (SCOM) by Paparella M. et al. ${ }^{1}$ One of the theories for its pathogenesis is the 'Continuum theory' as proposed by Paparella in which Otitis Media is described as a sequence of events, initiated by an insult that would lead to a cascade of events. It begins with OME, progresses through AOM and leads to COM and/ or its complications/sequelae. Patients with COM in one ear have a high chance of presenting with some degree of disease in the contralateral ear. The contralateral ear is the ear which is asymptomatic or the ear which has clearly less symptoms in the presence of an intact TM. Very few studies have evaluated this concept of silent COM. The aim of the study was to establish that patients with symptomatic unilateral COM can have subclinical/ asymptomatic otological findings suggestive of COM in the contralateral ear.

\section{Materials and Methods}

A cross-sectional observational study was conducted

1 - Department of ENT, Command Hospital(EC), Alipore, Kolkata.

Corresponding author:

Dr Ramakrishnan Narayanaswamy

email: drnramak5632@gmail.com 
in a multispecialty hospital in Eastern India from Jan 2015 to July 2016. All age groups were included. One hundred cases of strictly unilateral COM with or without cholesteatoma, with no history of otological complaints at all in the contralateral ear, were included in the study. Another 100 healthy subjects were taken from other non-otological patients in the ENT department for comparison, who served as control arm of the study. The findings which were reckoned to be of significance in the contralateral ear were retractions, myringosclerosis, thinning of TM and dimeric segments in TM, fluid/ glue, granulations, cholesterol granuloma, mucosal thickening and polyps in middle ear cleft, behind an intact tympanic membrane (SCOM). All the findings were recorded by the same investigator, to eliminate any subjective bias.

Detailed otorhinolaryngological examination including otoscopic, otoendoscopic and otomicroscopic examination were done. Pure tone audiometry and tympanometry were done. HRCT temporal bone was done in cases with clinical findings of squamous disease only, to evaluate bony erosion due to disease, impending extra or intracranial complications.

Patients of COM with previous ear surgery in contralateral ear or history of trauma to the contralateral temporal bone and patients with congenital malformations in the contralateral ear were excluded from the study.

Analysis of variance and correlation coefficient with significance levels was calculated on the data collected using appropriate statistical tests. Data was entered into a Microsoft Excel spreadsheet and then analyzed by SPSS 20.0.1 and Graph Pad Prism version 5. Data has been summarized as mean and standard deviation for numerical variables and count and percentages for categorical variables. The median and the interquartile range have been stated for numerical variables that are not normally distributed. Student's independent sample's t-test was applied to compare normally distributed numerical variables between groups; unpaired proportions were compared by Chi-square test or Fischer's exact test, as appropriate. Each of these statistics can be used to carry out either a one-tailed test or a two-tailed test. Once a t-value is determined, a p-value can be found using a table of values from
Student's t-distribution. If the calculated p-value is below the threshold chosen for statistical significance, then the null hypothesis is rejected in favour of the alternative hypothesis. $\mathrm{p}$-value $\leq 0.05$ was considered as statistically significant.

\section{Results}

Out of 100 cases of unilateral COM, 58 patients had some abnormality in the contralateral ear. Tympanosclerotic patches were the most common finding seen in 29 patients followed by tympanic membrane retractions seen in 28 patients. Many had multiple findings. (Table I). The age and sex distribution are as given at Table II and III respectively. Age and sex are not confounding factors.

Retraction of Pars tensa in the contralateral ear was statistically significant finding when compared between cases and control (Table IV) However, Attic retraction was found to be statistically significant when compared among the 2 subgroups (mucosal and squamous types of COM) $(6 \%)$ and controls (2\%). It was found to be more prevalent amongst squamous disease patients (Table V). Myringosclerosis patches were found in 35 contralateral ears in patients, whereas only 4 ears had TS patches amongst controls which was statistically significant. Dimeric segments were found in 21 ears in cases and only 4 ears in controls, which were statistically significant.

In 100 ears in the control group, only $11 \%$ had positive findings. TM retractions were seen in 5 subjects (2 Attic and 3 Pars tensa retractions). Myringosclerosis patches were seen in 4 subjects and dimeric TM in 4 subjects with 2 patients having multiple findings.

\section{Discussion}

Paparella et al. revealed that the disease in one ear is linked to subclinical disease in the contralateral ear. He validated the Continuum theory and described Silent COM. He studied microscopic changes in the temporal bones of contralateral ears. He professed that $\mathrm{COM}$ in one ear slowly affects the other ear too. ${ }^{1}$

Similarly, some other authors too conducted studies 
Table 1: Findings in the contralateral ear in cases.

\begin{tabular}{|c|c|}
\hline MUCOSAL DISEASE (85 PATIENTS) & SQUAMOUS DISEASE (15 PATIENTS) \\
\hline $\begin{array}{c}\text { Myringosclerosis patches (26 patients) (30.6\%) } \\
\text { TM retractions (19 patients) (22.4\%) }\end{array}$ & Myringosclerosis patches (3 patients) (20\%) \\
\hline $\begin{array}{c}\text { - Pars tensa retractions (17 patients) } \\
\text { - Attic retractions (2 patients) }\end{array}$ & - Pars tensa retractions (5 patients) \\
\hline Dimeric segments (18 patients) (21.2\%) & Dimeric segments (3 patients) (20\%) \\
\hline OME (2 patients) (2.4\%) & OME (1 patient) (6.7\%) \\
\hline
\end{tabular}

on this topic and came to similar conclusions regarding the affection of contralateral ear in cases of unilateral COM. Selaimen et al studied a total of 500 consecutive patients who had been diagnosed as having chronic otitis media with or without cholesteatoma.

They found that $376(75.2 \%)$ had abnormalities in the contralateral ear. ${ }^{4}$ Of 302 patients without Cholesteatoma $69.9 \%$ had abnormalities in the contralateral ear and of 198 patients with Cholesteatoma, 83.3\% had abnormalities in the contralateral ear. Between 2003 to 2009, Kayhan et al. did retrospective medical chart reviews of 412 patients and found a high prevalence of changes in contralateral ears, however in contrast to Selaimen's study, they found similar prevalence in mucosal and squamous groups. ${ }^{5}$ In 1996, Vartiainen et al. studied contralateral ears of 493 patients at the final follow up examination before surgery and found that in only $37.5 \%$ of the patients was the contralateral ear

Table II: Variables assessed vide the master chart (Contd.)

\begin{tabular}{|c|c|c|c|c|c|c|c|}
\hline $\begin{array}{l}\hat{b} \\
\frac{6}{0} \\
\frac{6}{b}\end{array}$ & $\sum_{\mathrm{Z}}^{\frac{0}{2}}$ & 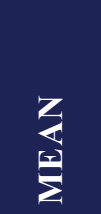 & $\theta$ & $\underset{z}{\underline{Z}}$ & $\underset{\sum}{\sum}$ & $\frac{Z}{\frac{Z}{2}}$ & $\begin{array}{l}\frac{6}{5} \\
\frac{1}{3} \\
\frac{1}{1} \\
\stackrel{1}{1}\end{array}$ \\
\hline Cases & 100 & 38.89 & 13.6943 & 12 & 76 & 39 & 0.1906 \\
\hline Controls & 100 & 36.44 & 12.6709 & 14 & 70 & 34 & \\
\hline
\end{tabular}

(Statistically not Significant) found to be normal, with atrophy of the pars tensa and tympanosclerosis being the most common abnormal findings followed by pars tensa and attic retractions.

The authors concluded that to improve the functional outcome of patients with chronic otitis media, earlier and more effective treatment of middle ear infections is needed. ${ }^{6}$ In 2002, Barbara et al. studied the contralateral ears of patients with unilateral COM in 108 patients and found in $46.3 \%$ of patients the contralateral ear was found to have some abnormality. ${ }^{7}$ Most common abnormal finding was retraction of tympanic membrane. In the study by Adhikari et al. out of 750 patients, in $68.4 \%$ of patients the contralateral ear was found to have some form of abnormality. ${ }^{8}$ Rosito et al. studied 85 pairs of temporal bones microscopically in cases of unilateral COM and found a high prevalence of changes $(91.8 \%)$ on the contralateral side. ${ }^{9}$

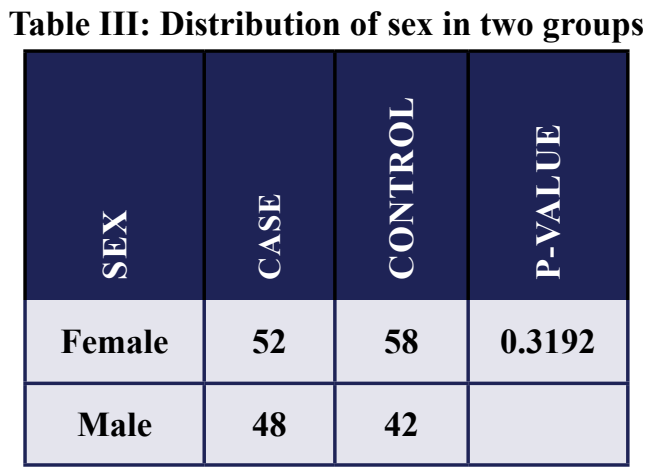

(Chi-square value: 0.9920; P-value: 0.3192, Statistically not significant) 
Table IV: Distribution of Pars tensa retractions (Sade ${ }^{2}$ grading) in contralateral ear in two groups

\begin{tabular}{|c|c|c|}
\hline & & \\
\hline No retraction & & 0 \\
\hline Grade 1 & 0 & 8 \\
\hline Grade 2 & 78 & 97 \\
\hline Grade 3 & 12 & 2 \\
\hline Grade 4 & 6 & 1 \\
\hline Total & 3 & 0 \\
\hline
\end{tabular}

(Chi-square value: 16.7771; P-value: 0.0021, Statistically Significant)

In a clinico-radiological study of the contralateral ear in acquired unilateral Cholesteatoma by Khalil et al. out of 50 patients with unilateral cholesteatoma and 25 control subjects with bilateral normal ears, $72 \%$ of the contralateral ears in cases showed signs of retraction pockets, granulation tissue or chronicity. ${ }^{10}$ Ahmed et al. found abnormalities in $37 \%$ of the contralateral ears of 46 patients with unilateral COM. ${ }^{11}$ All these studies by different authors from around the world are consistent

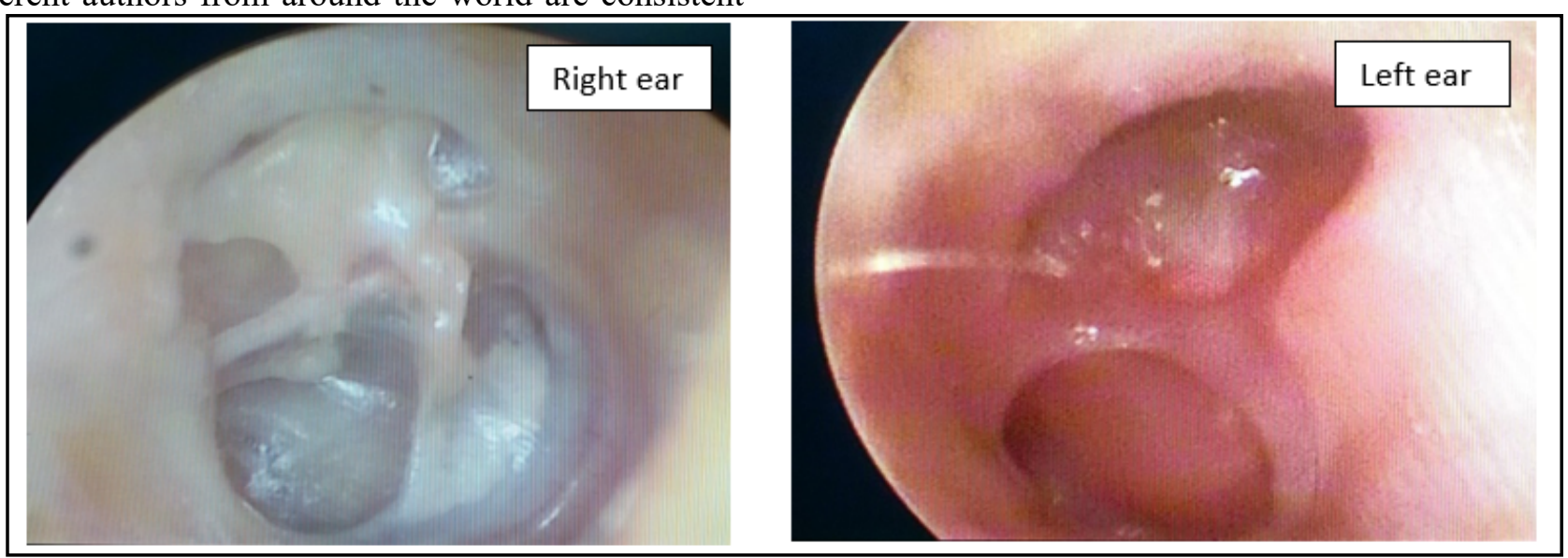

Fig. 1. Changes in the contralateral right ear are clearly evident in this picture with exposure of ossicles in the attic due to scutum erosion (Tos ${ }^{3}$ grading) in two subgroups in cases and controls

\begin{tabular}{|c|c|c|c|}
\hline & & & \\
\hline & 2 & 2 & 0 \\
0 & 0 & 0 & 0 \\
0 & 0 & 0 \\
\hline No retraction & 83 & 11 & 98 \\
\hline Grade 1 & 2 & 0 & 0 \\
\hline Grade 2 & 0 & 0 & 0 \\
\hline Grade 3 & 0 & 4 & 2 \\
\hline Total & 2 & 4 & 2 \\
\hline
\end{tabular}

(Chi-square value: 15.9783; P-value: 0.0003, Statistically Significant)

with findings in our study.

The factors that affect eustachian tube functions on one side frequently have effect on the contralateral side too. (Figs. 1 and 2) We can thus infer that the disease of $\mathrm{COM}$ in one ear affects the contralateral ear slowly and silently. Hence both ears can be affected as a pair and a slow pathological process continues behind an intact TM and kept hidden from the view, unless it is actively sought for.

Table V: Distribution of contralateral attic retractions 


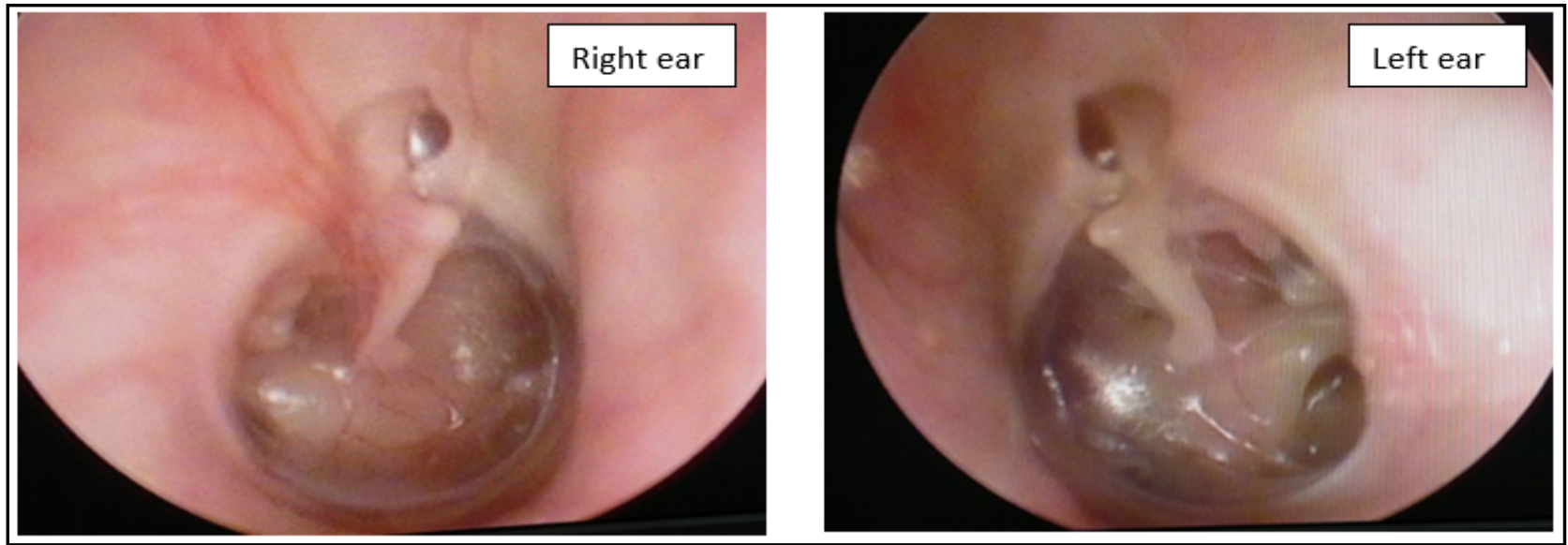

Fig. 2. Both ears showing scutum erosion and a thin TM plastered to promontory. In this case of Inactive Squamous disease, both ears have been affected to more or less equal extent. To begin with, patient presented with Inactive squamous disease (Left).

The changes seen in the contralateral ear applies more to patients with COM squamous disease, as these patients have more prevalence of abnormal findings including attic retractions. In one study the authors found improvement in the contralateral ear after operative treatment of the ear with COM. ${ }^{12}$

This is one step ahead in proving this theory. Hence the clinicians should treat not only the affected ear in COM but also need to follow up the contralateral ear very cautiously. This will go a long way in preserving the already compromised hearing of the patients and improving his/her quality of life.

\section{Conclusion}

Chronic Otitis media is a disease with very varied clinical presentation and disease in one ear has been sometimes found to be associated with subtle to gross changes without any symptoms, observed in the contralateral ear. These changes can eventually result in beginning of a gradual and chronic inflammatory process in the contralateral ear.

Therefore, it is imperative to exert caution in the clinical assessment of the contralateral ear. The contralateral ear warrants due respect and reckoning with regular follow-up.

\section{References}

1. Paparella M. Silent otitis media. Laryngoscope 1980; $90(7 \mathrm{Pt}$. 1): 1089-98

2. Sade J, Avraham S, Brown M. Atelectasis, retraction pockets and cholesteatoma. Acta Otolaryngol. 1981;92:501-12

3. Tos M, Poulsen G. Attic retractions following secretory otitis. Acta Otolaryngol. 1980;89:479-86

4. Selaimen S, Rosito L P S, Dornelles C. Contralateral ear in Chronic Otitis media: A histologic study. Archives of Otolaryngology: Head and Neck surgery 2008; 134(3): 290-3

5. Kayhan F T, Sayin I. Chronic otitis media-evaluation of the contralateral ear. KBB-Forum 2011; 10 (4)

6. Vartiainen E, Kansanen M, Vartiainen J. The contralateral ear in patients with chronic otitis media. Am J Otol. 1996; 17(2): 190-2

7. Barbara A. Contralateral ear in chronic otitis media. Otorrinolaringol. [online] 2002; 68(2): 245-9

8. Adhikari P, Khanal S. Status of contralateral ear in patients with chronic otitis media. The Internet Journal of Health 2009; 10(2)

9. Rosito LPS, da Costa SS, Schachern PA, Dornelles C et al. Contralateral ear in chronic otitis media: a histologic study. Laryngoscope 2007; 117(10): 1809-14

10. Khalil H, Saleh MA. A clinico-radiological study of the contralateral ear in acquired unilateral cholesteatoma. Rev Laryngol Otol Rhinol. (Bord) 2004; 125 (1): 17-22

11. Ahmed K E, Elhamd A, Sayed R H, Moussa A E. The other ear in unilateral chronic suppurative otitis media. Saudi Journal of Oto-Rhino-Laryngology Head and Neck Surgery 2007; 9(1): 24-6

12. Jadia S, Mourya A. Pre and Post Operative Status of Contralateral Ear in Unilateral Chronic Otitis Media. Indian J Otolaryngol Head Neck Surg. 2016; 68: 20. 